\title{
Radial velocities of five globular clusters obtained with AAOmega
}

\author{
P. Székely ${ }^{1,2, \star}$, L. L. Kiss ${ }^{3}$, K. Szatmáry ${ }^{1}$, B. Csák ${ }^{4}$, G. Á. Bakos ${ }^{5}$, and T. R. Bedding ${ }^{3}$ \\ 1 Department of Experimental Physics \& Astronomical Observatory, University of Szeged, H-6720 Szeged, Dóm tér 9., \\ Hungary \\ ${ }^{2}$ Hungarian Eötvös Fellowship, School of Physics, University of Sydney, NSW 2006 Australia \\ 3 School of Physics, University of Sydney 2006, NSW 2006 Australia \\ 4 Department of Optics and Quantum Electronics, University of Szeged, H-6720 Szeged, Dóm tér 9., Hungary \\ ${ }^{5}$ Harvard-Smithsonian Center for Astrophysics, Cambridge, MA, USA
}

The dates of receipt and acceptance should be inserted later

Key words globular clusters - Galaxy: kinematics and dynamics - Galaxy: halo

\begin{abstract}
Using the recently commissioned multi-object spectrograph AAOmega on the 3.9m AAT we have obtained mediumresolution near-infrared spectra for 10,500 stars in and around five southern globular clusters. The targets were 47 Tuc, M12, M30, M55 and NGC 288. We have measured radial velocities to $\pm 1 \mathrm{~km} \mathrm{~s}^{-1}$ with the cross correlation method and estimated metallicity, effective temperature, surface gravity and rotational velocity for each star by fitting synthetic model spectra. An analysis of the velocity maps and velocity dispersion of member stars revealed systemic rotation in four of the target clusters.
\end{abstract}

(C) 2007 WILEY-VCH Verlag GmbH \& Co. KGaA, Weinheim

\section{Introduction}

Globular clusters are among the oldest objects in our Galaxy and their stars provide unique information on Galactic evolution through cosmic times. These clusters are sensitive indicators of the Galactic gravitational potential because the velocity distribution of the stars in their outskirts is affected by the ambient field of the Milky Way. They are believed to have undergone substantial dynamical evolution, which is affected by processes responsible for the "evaporation" of stars (Meylan \& Heggie 1997): tidal interaction with the Galaxy and two-body relaxation. In most of the cases the cluster moves around the Galactic center along very elongated orbit. Close to the perigalactic position the cluster suffers the strongest gravitational interactions which can result in tidal shocks and lead to the formation of tidal tails. Recent investigations have found long tidal tails in two lowconcentration clusters, Palomar 5 and NGC 5466, thus confirming theoretical predictions (Odenkirchen et al. 2001; Grillmair \& Johnson 2006). The success behind these results was secured by deep photometric surveys, most notably the SDSS project, which allowed cluster membership determination with statistical analysis of colors and magnitudes of stars. Another possibility is offered by spectroscopic observations of individual stars in order to derive metallicity and radial velocity, both efficient marker of cluster members.

AAOmega, the newly commissioned multi-object spectrograph on the 3.9 meter Anglo-Australian Telescope provides an excellent opportunity to measure up to 350-360 ra-

\footnotetext{
^ Corresponding author: e-mail: pierre@physx.u-szeged.hu
}

dial velocities with a single exposure. Due to its large field of view ( 2 degrees) it can observe stars which are located far from the cluster in the plane of the sky. With a velocimetric accuracy of about 1-2 $\mathrm{km} \mathrm{s}^{-1}$, one can easily identify escaped stars which have the same or very similar radial velocities as the host cluster.

In this paper we present an analysis of radial velocities of cluster member stars in terms of systemic rotation for five southern globular clusters. A detailed investigation of their possible tidal tails has been reported by Kiss et al. (2007).

\section{Observations and data reduction}

The targets were selected from the globular cluster catalogue of Harris (1996) based on the following criteria: we preferred nearby, unreddened clusters with large radial velocities in respect to the Galactic field containing mostly disk stars. The latter is very useful to distinguish cluster member stars. We also considered recent results on tidal tails (in case of NGC 288; Leon et al. 2000), interesting dynamical history (M12; de Marchi et al. 2006) or internal structures (47 Tuc; Meylan \& Mayor 1986) to choose targets. We ended up with the following clusters: 47 Tuc, NGC 288, M12, M30, and M55. By chance we also recorded radial velocities of a few stars in the extragalactic globular cluster NGC 121 that belongs to the Small Magellanic Cloud.

Our observations were carried out on 7 nights in August, 2006. Throughout the run the seeing stayed between 1.2 and 2.2 arcsec. In total we took spectra for more than 10,500 stars using the D1700 grating, recording near-infrared spec- 
Table 1 Total number of stars identified as members, cluster radial velocity from Harris (1996) and the measured mean velocity.

\begin{tabular}{lcrr}
\hline \hline Cluster & $\begin{array}{c}\text { No. } \\
\text { stars }\end{array}$ & $\begin{array}{r}v_{\text {rad }}(\mathrm{H} 96) \\
\mathrm{km} \mathrm{s}^{-1}\end{array}$ & $\begin{array}{r}\left\langle v_{\text {rad }}\right\rangle \\
\mathrm{km} \mathrm{s}^{-1}\end{array}$ \\
\hline 47 Tuc (NGC 104) & 911 & -18.7 & -16.1 \\
M12 (NGC 6218) & 158 & -42.2 & -40.4 \\
M30 (NGC 7099) & 129 & -181.9 & -178.1 \\
M55 (NGC 6809) & 433 & 174.8 & 171.7 \\
NGC 288 & 123 & -46.6 & -43.5 \\
\hline
\end{tabular}

tra centered on the Ca II triplet lines. The spectra ranged from $8350 \AA$ to $8790 \AA$ with a resolving power $\lambda / \Delta \lambda=$ 10,500 . For each cluster we observed several fields centered on the cluster, containing 300-350 stars per configuration. To reach a signal-to-noise ratio between 50 and 250 we exposed 60 to 90 minutes in total. For sky background measurements and guiding we used 30-40 fibers per configuration.

The target stars were selected from the 2MASS point source catalogue (Skrutskie et al., 2006). We fitted a line to the Red Giant Branch (RGB) of each cluster and selected stars which matched its color and magnitude. For M12 and 47 Tuc we selected the lower part of the RGB. Due to the sparse field star population around M30 and NGC 288, we did not filtered their stars, but used the whole field. The full magnitude range of the target stars in $K$-band was 7 mag (from 8 mag to $15 \mathrm{mag}$ ) but for a single configuration field we limited the brightness range to 3 mag in order to avoid cross-talk between the fibers due to scattered light.

The spectra were reduced with the standard $2 \mathrm{dF}$ data processing pipeline (drcontrol), which extracts automatically the wavelength calibrated spectra. Continuum normalization was done with the IRAF task onedspec.continuum. After this we cleaned the spectra of the remnants of the strongest skylines via linear interpolation of the neighboring continuum.

For this study we derived two parameters for each star based on its spectrum: radial velocity and the full equivalent width of the Ca II triplet lines $\left(\Sigma E W=W_{8498}+\right.$ $\left.W_{8542}+W_{8662}\right)$. The latter was determined by fitting a sum of a Lorentzian and a Gaussian to the line profiles (Cole et al. 2004). Radial velocities were determined in an iterative way. An initial velocity estimate was provided by the line profile fit, which was used to find the best-fit spectrum in the extensive spectrum library of Munari et al. (2005). The fitted model spectrum was then cross-correlated with the observed one, giving the finally adopted velocity. The estimated accuracy is about $\pm 1-2 \mathrm{~km} \mathrm{~s}^{-1}$.

${ }^{1}$ IRAF is distributed by the National Optical Astronomy Observatories, which are operated by the Association of Universities for Research in Astronomy, Inc., under cooperative agreement with the National Science Foundation.

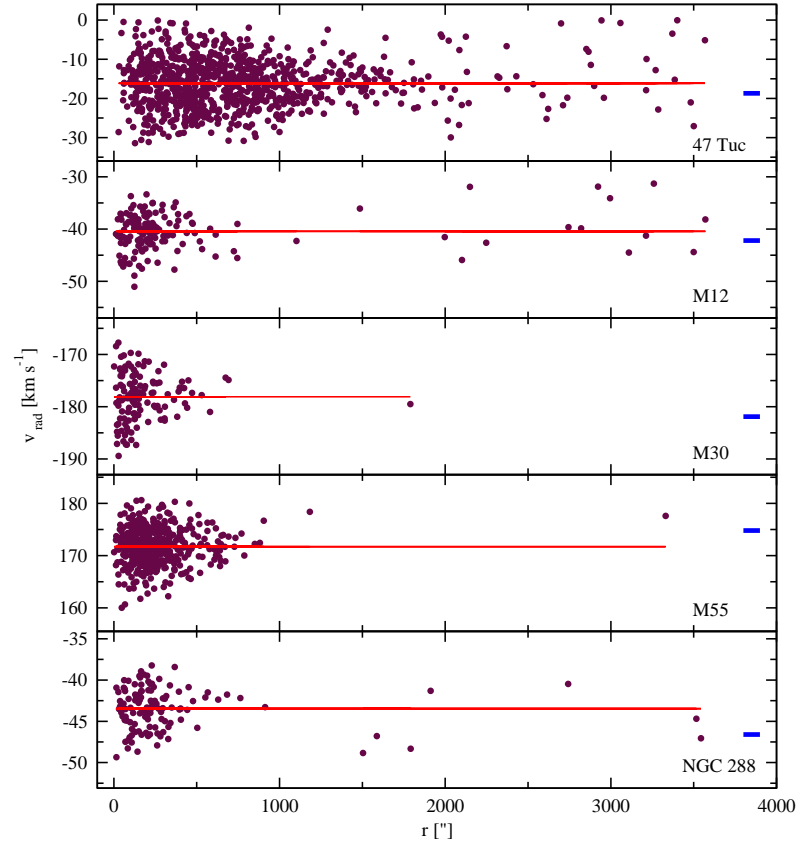

Fig. 1 Heliocentric radial velocities of the member stars versus distance from the cluster center. The horizontal lines show the mean cluster velocities. Thick lines indicate the catalogued values from Harris (1996). The wide distribution of the velocities around the mean values is partly due to the intrinsic velocity distribution of cluster stars.

\section{Results}

We identified cluster members using the radial velocities, equivalent widths and $K$ magnitudes. The selection was based on the $v_{\text {rad }}$ vs. $\Sigma E W$ and $\Sigma E W$ vs. $K$ magnitude correlations and led to the identifications of 123-911 stars as members in the five clusters (see Table 1). Note that these sets are more constrained than those of Kiss et al. (2007), who only used the radial velocity to determine cluster membership.

First we plot heliocentric radial velocities of the member stars as function of distance from the cluster center in Fig.1. Here the horizontal lines represent the cluster mean radial velocities, while on the right-hand side of the plot thick lines mark the catalogued values from Harris (1996). While there are differences up to $3 \mathrm{~km} \mathrm{~s}^{-1}$ in the measured means and the catalogued values, our samples are more extensive than any previous ones for all clusters except 47 Tuc, which may explain most of the differences. Because of the excellent statistics, Fig. 10 is clearly dominated by the characteristic wedge-shaped velocity distribution that can be used to derive the velocity dispersion profile, which is a sensitive indicator of the dynamical properties.

In Fig. 2. we plot celestial positions of member stars for 47 Tuc and M55, color-coded by their radial velocities. It is very prominent that there is a well-defined asymmetry in both clusters, which can be interpreted as result of rotation. 

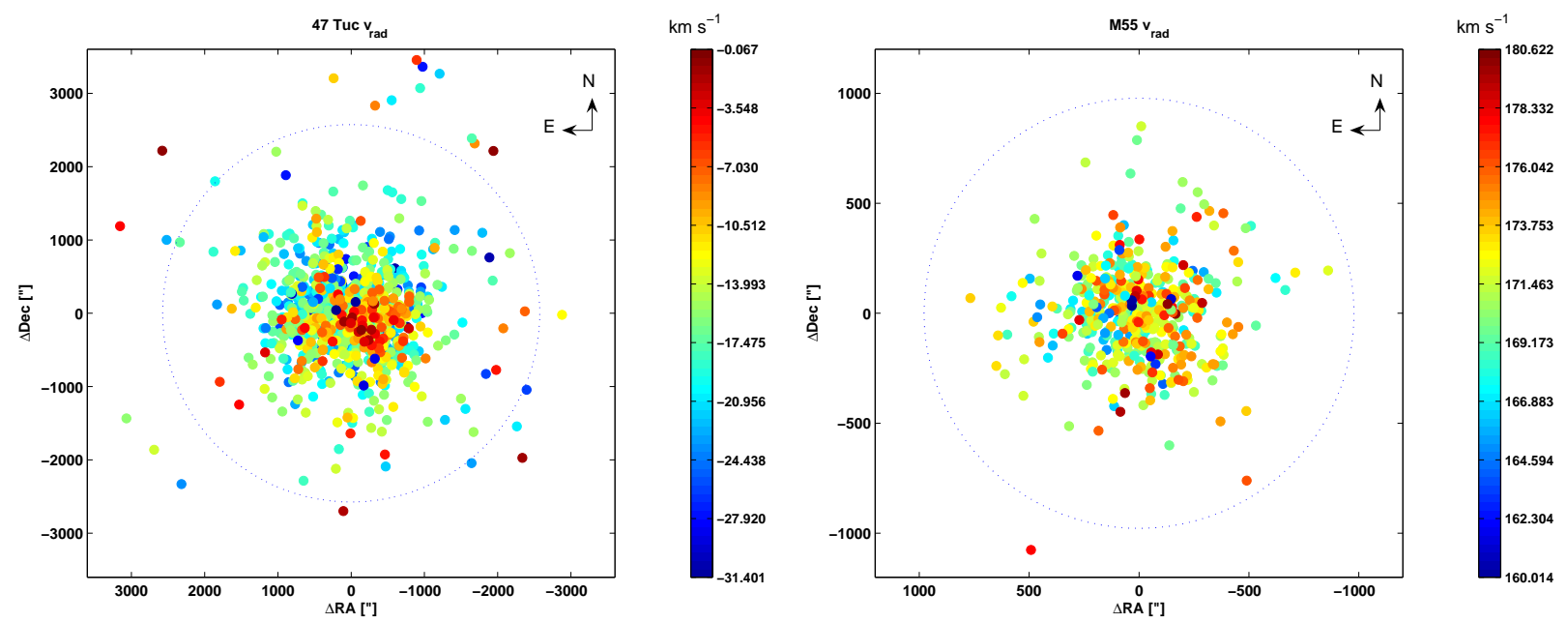

Fig. 2 Left panel: stellar positions in 47 Tuc with radial velocities encoded in colors. The circle shows the tidal radius $\left(r_{t}=42.9^{\prime}\right)$. Right panel: the same for M55 $\left(r_{t}=16.3^{\prime}\right)$. Note the smaller field of view.
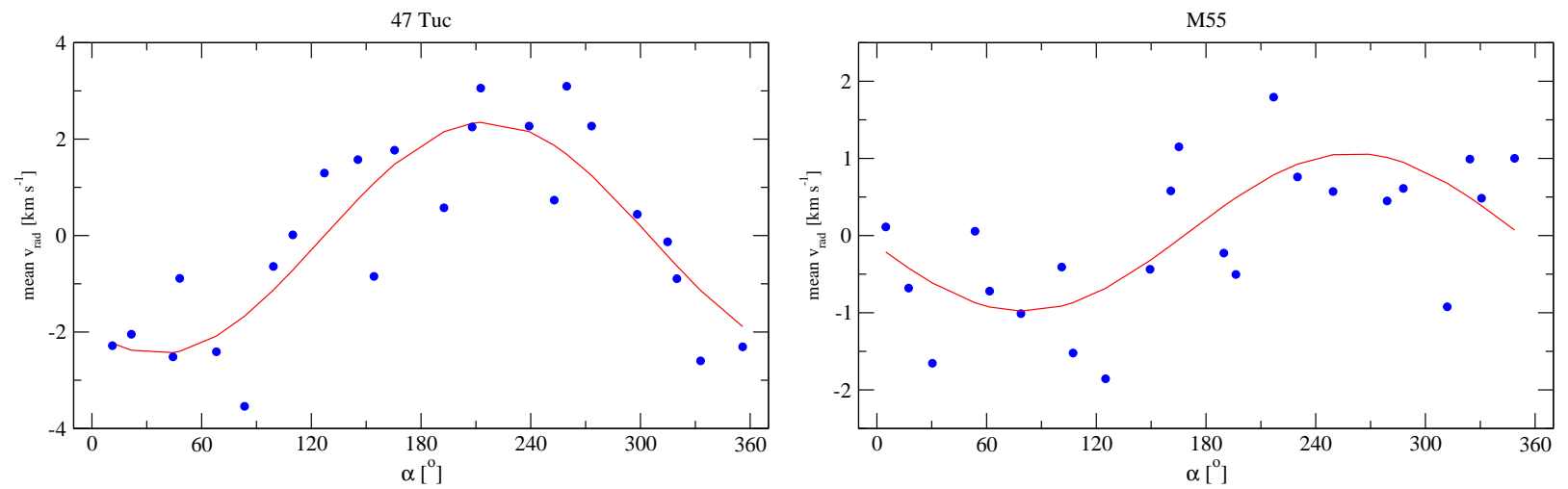

Fig. 3 Mean radial velocities versus polar angle in 24 azimuthal bins with equal width. We also plotted the best-fit sine curves.

To find quantitative parameters of the systemic rotation in these two clusters, we plotted the mean radial velocities versus position angle in Fig. 3. The effects of the internal velocity dispersion were minimized by binning the velocities in 15 degree-wide sectors of the position angles $\left(\alpha=0^{\circ}\right.$ corresponding to North, $90^{\circ}$ to East). The mean cluster velocities listed in Table 1 were subtracted for clarity. The sinusoidal shape of the distributions is well recognizable in both cases, though it is much clearer for 47 Tuc. For the other three clusters similar plots did not reveal obvious patterns.

Another useful method to detect azimuthal dependence of the radial velocities is stepping an imaginary axis through the globular cluster in small angular increments (e.g. one degree) and calculate the differences of the mean or median radial velocities on both sides of the axis (e.g. Côté et al. 1995). The resulting curves are plotted in Fig. 4, Based on this we can put an upper limit to the rotational velocity projected into the line of sight for each cluster by considering the amplitude of the curve. For 47 Tuc, the rotational velocity is $6.6 \mathrm{~km} \mathrm{~s}^{-1}$, being in perfect agreement with the results of Meylan \& Mayor (1986) and Anderson \& King (2003).
Table 2 Parameters of the detected systemic rotations: A is the peak-to-peak velocity amplitude, while the position angle of the rotational axis is given in both equatorial and galactic coordinates.

\begin{tabular}{cccc}
\hline \hline Cluster & $\begin{array}{c}\mathrm{A} \\
\mathrm{km} \mathrm{s}^{-1}\end{array}$ & $\begin{array}{c}\text { PA (eq.) } \\
\circ\end{array}$ & $\begin{array}{c}\text { PA (gal.) } \\
\circ\end{array}$ \\
\hline 47 Tuc & $6.6 \pm 0.07$ & $130 / 310 \pm 5$ & $122 / 302 \pm 5$ \\
M12 & - & - & - \\
M30 & $1.5 \pm 0.3$ & $16 / 196 \pm 15$ & $90 / 270 \pm 15$ \\
M55 & $2.4 \pm 0.1$ & $170 / 350 \pm 7$ & $61 / 241 \pm 7$ \\
NGC 288 & $1.4 \pm 0.15$ & $97 / 277 \pm 10$ & $52 / 232 \pm 10$ \\
\hline
\end{tabular}

The rotational velocity estimates for the other three clusters are listed in the second column of Table 2 For M12, the curve is clearly non-sinusoidal, so that we did not attempt to fit the data.

To derive the orientations of the projected rotational axis in the plane of the sky we fitted a sine wave to the curves presented in Fig. 4. The results are listed in Table 2 . We also converted the equatorial position angles to galactic coordi- 


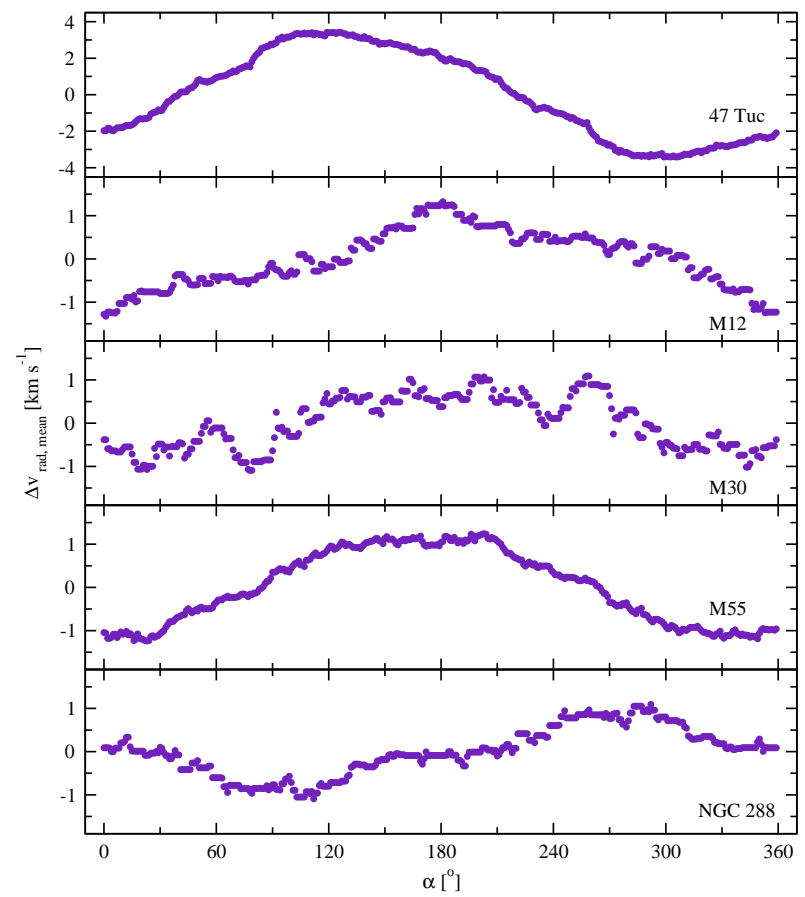

Fig. 4 Mean radial velocities on both sides of an imaginary axis stepped through the clusters. Note the different scale on the $y$ axis in case of 47 Tuc.

nates to see if there is a pattern in the directions. Indeed, for the four clear detections of systemic rotation we find directions being close (within \pm 20 - $25 \mathrm{deg}$ ) to parallel with the Galactic plane, which might be an evidence of non-random distribution of rotational axes, but the size of this sample is too small even for some statictical approaches. In any case, we confirmed rotation in 47 Tuc and discovered it in M30, M55 and NGC 288. For M12 we will have to clean further the sample of the Galactic field contamination before a new attempt at detecting rotation is considered.

\section{Future work}

Our data represent a unique source of information on the studied five globular clusters. We will separate cluster members from the Galactic field stars by detecting clumpiness in the full parameter space of the physical parameters, including radial velocity, metallicity, effective temperature, surface gravity. Global kinematics, mass-to-light ratios and star formation histories will be constrained from the cleaned samples of member stars. We will be particularly interested in the age-metallicity relation because a spread in metallicity can be a sign of prolonged star formation over 2-4 Gyrs (Stanford et al. 2006).

We are specifically interested in investigating mechanisms that affect velocity distributions in globular clusters and, in particular, the tidal tails. Theories to be tested include tidal heating of the evaporated stars by the external gravitational field (Drukier et al. 1998), the presence of a dark matter halo around the clusters (Carraro \& Lia 2000), and a breakdown of the Newtonian dynamics in the weakacceleration regime (Scarpa et al. 2007). The latter hypothesis is particularly interesting because modified Newtonian dynamics, valid for accelerations below $a_{0} \sim 1.2 \times 10^{-8}$ $\mathrm{cm} \mathrm{s}^{-2}$, may offer an alternative to the dark matter, with far-reaching implications for cosmology.

For each cluster we aim to measure the velocity dispersion profile of stars, which shows a characteristic decrease and flattening at distances from the cluster centre (Scarpa et al. 2007). Globular clusters are relatively simple stellar systems, with each star's motion dictated by the Newtonian gravitational pull of the other cluster members. However, any departure from the classical laws of dynamics (such as Modified Newtonian Dynamics: MOND, originally introduced by Milgrom 1983) predicts that the flattening should occur at the same absolute acceleration regardless of the Galactic environment of the cluster. Hence, we will characterize the dynamical properties of the clusters as a fundamental probe of Newtonian gravity. Since rotation of the cluster can introduce systematic errors in the interpretation, we will subtract a smoothed velocity field from the data. The residuals will show whether the velocity dispersion decreases at large radii and whether it reverses to an increase outside the tidal radius. To disentangle possible breakdown of the Newtonian dynamics and conventional dynamic effects such as tidal heating, one has to observe clusters of very different parameters, including different Galactocentric distances and determine the actual acceleration at which the dispersion profile flattens. This presented sample is a good starting point in this direction and while much effort will be devoted to model the cluster dynamics, we also plan to extend the observational data base with further clusters.

Acknowledgements. This project has been supported by the Hungarian OTKA Grant \#T042509, a Hungarian Eötvös Fellowship to PSz and the Australian Research Council. LLK is supported by a University of Sydney Postdoctoral Research Fellowship. Support for program number HST-HF-01170.01-A to G.Á.B. was provided by NASA through a Hubble Fellowship grant from the Space Telescope Science Institute, which is operated by the AURA, Inc., under NASA contract NAS526555. G.Á.B. also wishes to thank useful discussions to A. Pál. We are very grateful to the staff of the Anglo-Australian Observatory for their kind and helpful support during our observing run.

\section{References}

Anderson, J., King, I. R., 2003, AJ, 126, 772

Carraro, G., Lia, C., 2000, A\&A, 357, 977

Cole, A. A., et al., 2004, MNRAS, 347, 367

Côté, P., et al., 1995, AJ, 454, 788

de Marchi, G., Pulone, L., Paresce, F., 2006, A\&A, 449, 161

Drukier, G.A., et al., 1998, AJ, 115, 708

Grillmair, C. J., Johnson, R., 2006, ApJ, 639, L17

Harris, W. E., 1996, AJ, 112, 1487

Kiss, L. L., et al., 2007, ApJL, in press (astro-ph/0703247)

Leon, S., Meylan, G., Combes, F., 2000, A\&A, 359, 907

Meylan, G., Mayor, M., 1986, A\&A, 166, 122 
Meylan, G., Heggie, D. C., 1997, A\&ARv, 8, 1

Milgrom, M., 1983, ApJ, 270, 365

Odenkirchen, M., et al., 2001, ApJ, 548, L165

Munari, U., et al., 2005, A\&A, 442, 1127

Scarpa, R., et al., 2007, A\&A, 462, L9

Skrutskie, M. F., et al., 2006, AJ, 131, 1163

Stanford, L. M., et al., 2006, ApJ, 647, 1075 